\title{
Structural assessment of remodelled shells of Heinz Isler
}

\author{
Abtin Baghdadi ${ }^{1} \cdot$ Mahmoud Heristchian $^{2}\left(\mathbb{0} \cdot\right.$ Harald Kloft $^{1}$
}

Received: 29 June 2018 / Accepted: 17 October 2019 / Published online: 2 November 2019

(c) The Author(s) 2019

\begin{abstract}
Heinz Isler as the most famous contemporary shell designer has widely employed physical pre-modelling techniques for construction of many concrete shell structures. Through the physical approach to optimal form finding, Isler accomplished shell structures with robust performance. It would be interesting and beneficial to re-assess Isler's shells, hence, this article attempts to study the structural performance of eight notable shells of Isler. Through reverse engineering and by the assistance of Rhino, MATLAB and Grasshopper, the precise geometry of Isler's selected shells were modelled for the finite element analysis under their self-weight. The structural analysis was performed, with the parallel use of finite element software SAP2000 and Abaqus. The identical results of the two packages, further confirmed the accuracy of the analysis. The essential properties of various forms of the shells and their differences in behaviour were pinpointed and discussed within the calculations and the results were compared with the data of the genuine published references on Isler's works. The internal forces, the amount of von Mises stresses, support reactions and the buckling loads of the shells are explored. The analyses revealed that, despite of their major membrane action, all the shells had negligible amount of bending moments, especially near the supports. However, in general, all the shells exhibited an appropriate performance under the applied actions. But, at the same time, they exhibited different buckling behaviour as a probable source of instability in them.
\end{abstract}

Keywords Shell structure $\cdot$ Heinz Isler $\cdot$ Reverse engineering $\cdot$ Finite element analysis $\cdot$ Optimal performance

\section{Introduction}

This section gives a brief account of some general aspects of shells and reviews some historically significant breakthroughs in calculation of shell structures. Insertion of a curvature in a plate $(=$ a flat shell $)$ under transverse loads, reduces its flexural actions and increases its normal actions, where the amount of reduction in flexural action is directly related to the nature and "magnitude" of its curvature. The height-to-span ratio is a simple measure of the curvature of shells which reflects the structural and economic performance of a shell as well. The optimal range of this ratio for the arches is [0.25, 0.30], (Heristchian et al. 2014). This ratio remains unchanged for various situations, furthermore, it has

Mahmoud Heristchian

heris@azad.ac.ir

1 Department of Architecture Civil Engineering and Environmental Sciences, Institute of Structural Design, TU Braunschweig, Braunschweig, Germany

2 Department of civil engineering, South Tehran Branch, Islamic Azad University, Tehran, Iran intrinsic relation with the material properties of the arch, and with its internal forces i.e., shear $(V)$, bending moment $(M)$ and normal force $(F)$. Calculating of pressure line for an arch under a specific applied load on its axis and reducing the eccentricity $(e=M / F)$ of its typical sections to zero is ideal for the concrete arches that are weak in tension. For this reason, the central coordinate of the economical and favourable concrete arches matches its pressure line (Akhavan and Tahooni 1996). At the same time, that is also, the main parameter in optimization of arches and shells. Finding the pressure area of shells is a challenging problem because of simultaneous interaction of its form with the loads and possible support locations in 3D space. Due to the complexity of the shell geometries, historically, the trials were usually restricted to the exactly calculated simple shapes such as the shapes proposed by Timoshenko and Woinowsky-Krieger (1959), etc. Some methods of calculation of shells, such as differential methods and the methods practiced by Isler are discussed in the section devoted to Isler's work.

However, few structural engineers consider mathematical points in working on more accurate geometries towards more optimal shells like hyperbolic paraboloid shapes designed by 
Felix Candela (Sprague 2013). In their studies, the type of the shell and its symmetries were usually the most important factors in calculations. However, the shell designers usually have a poetic overtone look for free forms that are unachievable with the exact feasible calculations. On the other hand, another practical method for finding the shell forms without intensive calculations is the method of mimicking natural shapes which have found its place in a wide range of usages. Mimicking natural structural shapes has two advantageous reasons which encouraged the designers to follow this simple method: (1) selection of the shape by hanging and finding its geometry according to the effect of weight and gravity which forms a shape under pure tension. (2) The behaviour of these structures can be scaled down independent of their actual and final size. This means that making and testing small physical models can be a quick and practical method. Under prescribed boundary conditions, once flexible structural shell form gets inverted, due to its self-weight it attains the optimal and economic shape under pure tension. A form obtained in such a way, under the reverse loading will be in pure compression. In this method, the rigidity of the material under test, plays an important role. The Galileo Galilei's (1564-1642) concept of "square-cube law" states that the area increases with the square of the scale factor, and the volume (hence the mass) increases with the cube of the scale factor. Simon Stevin (1548-1620) was one of the earliest developers of the force vectors based on the mathematical representation. Byvough, in 1586, published "The Principles of the Art of Weighing", which described the parallelogram of forces based on the models of funicular (2D, 3D) shapes. Later, Robert Hooke (1635-1703) with Christopher Wren (1632-1723) designed a Cathedral in London using the inverted catenary models. Christopher Wren sketched a 33-m diameter dome, showing usage of suspended chain method in design of buildings. That is one of the well-known and satisfactory methods of employing physical models to obtain structures resistant to compression, which increased Hooke and Wren's confidence in this method. In evaluation of 100-year-old, 50-m diameter dome, Giovanni Poleni (1683-1761) employed Hooke's method in Rome, however, minor cracks formed in the dome (Adriaenssens et al. 2014). Considering Galileo's law of size effect, construction of a $50 \mathrm{~m}$ dome should have presented a challenging construction problem, for its time.

Michelangelo (1475-1564) as an architect and engineer reduced the weight of the structure using the method of double skin shell, suggested by Filippo Brunelleschi (1377-1446). Later in 1580s, to decrease the outward thrusts, Giambattista Della Porta (1535-1615) added the height-to-span ratio of the domes by extending their lower parts. This was followed by 2- and 3-dimensional arches used by Friedrich Goesling (1837-1899) in a number of models in the 1890s. The method was further developed by Antoni Gaudi (1852-1926) who employed suspended bags from strings of the models of several masonry shells (González et al. 2018). Frei Otto (1925-2015) an army pilot looking for a shelter started using models made by cable networks and other membrane elements to determine the tension in cable networks, during the times that practical use of computers for this purpose was rather unfeasible (Chilton and Chuang 2017). Finally, Heinz Isler (1926-2009) as the most famous concrete shell builder, in twentieth century, designed and built several complex and stable shells in Europe by the method similar to Hooke's method. In one of his techniques, Isler created the forms by free hanging of the soaked fabric, then, to increase the stiffness of the shell, he folded its edges based on his own experience. Furthermore, he hardened the models, either by freezing them outdoors in cold weather or by adding cement mixture. Finally, he scaled them up and reproduced their funicular geometry (Brew and Lewis 2007). Isler also used simple calculations, based on the assumption that there is no bending moment, to estimate the amount of forces, however a negligible flexural action exists near the supports. Additionally, he presented the buckling equation in a colloquium in 1982 (Chilton and Isler 2000).

\section{Isler's models and analysis}

The exceptional performance of Isler's shells within 4-5 decades, and the importance of his approach to modelling, has encouraged many designers to re-evaluate his shells and get inspired by them.

As an example, the Naturtheater Groetzingen shell (1977) with $42 \mathrm{~m}$ by $28 \mathrm{~m}$ span and $10 \mathrm{~m}$ height, covering an area of approximately $650 \mathrm{~m}^{2}$ and with 220 Tons of reinforced concrete, was simulated in SAP2000 four decades after its construction time, at Princeton University (Maurer et al. 2013). According to the results of analysis, the maximum tensile force was $1152 \mathrm{kN} / \mathrm{m}$, which occurred mainly in the supports and near the edges of the shell, and it was less than the half of maximum compressive force amounting to $2785 \mathrm{kN} / \mathrm{m}$. The evaluation model of the structure in Norwich Sports Park used 3D scans of pre-models and simulated it within the finite element software called NURBS (Borgart and Eigenraam 2012). This model confirmed that along the curvature, the distribution of the bending moment changes and increases between the top part and the long edges of the shell, which shows the effect of edge stiffeners. The numerical studies continued by working on the Deitingen Service Station, Coop Distribution Centre, Buergi Garden Centre and on other Isler's shells (Brew and Lewis 2007; Ramm and Mehlhorn 1991; Neuhäuser 2014; Emami 2015). Despite of the above-mentioned case studies, Isler's shells deserve further study to clarify the influence of the utilized method of form finding on the structural characteristics of the shells, 



Fig. 1 Shells mesh-rebar pattern

and to evaluate their structural and buckling features comprehensively and compare with each other.

\section{Reconstruction of shells}

\section{Software and material}

This paper has modelled and studied Isler's eight shells in the finite element software, Abaqus, as a homogeneous section together with a composite layer to allow for assignment of re-bars of the reinforced concrete material. The Static General method of analysis was employed. The models have been evaluated without using Interaction modulus under their self-load. In parallel to Abaqus modelling, also, SAP2000 was used with the same linear material properties and definitions. The mechanical properties of the concrete material of Isler's shell constructions are not identical and different references have mentioned different values for it. The typical concrete mixture of Isler's shells constitutes of cement percentage of maximum $\left(325 \mathrm{~kg} / \mathrm{m}^{3}\right)$, gravel stone with a maximum dimension of $(15 \mathrm{~mm})$ (Christian and David 2012) for the concrete of $28 \mathrm{MPa}$ (C-28) compression strength. Since, the aim of this study is to compare the structural performance of various shells with each other, thus, in all of the shells, the same strength and material properties for concrete is assumed. The ratio of steel to concrete material by weight is $\left(\frac{15}{220}\right)$, (Maurer et al. 2013). The mesh of steel reinforcement is arranged symmetrically on both sides of the shell Fig. 1. The mechanical properties of the re-bars are assumed to be according to the standard ASTM A992 (with yield and ultimate stresses $F_{\mathrm{y}}=35153.5$ and $\left.F_{\mathrm{u}}=45,700 \mathrm{t} / \mathrm{m}^{2}\right)$ as the most available steel types. Nonetheless, other considerations and addition of structural elements, utilized by Isler in reducing the tension and increasing the buckling factor, such as using post-tensioned strands between the supports, influence the structural performance of the shells, however, since the focus of the current study is just on a comparison between the forms of the shells, these details were not considered here.

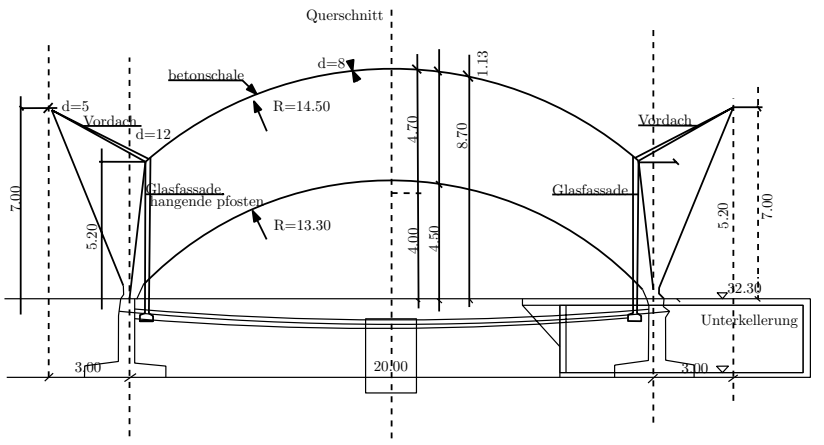

Fig. 2 Garten Haus Wyss elevation (Stefan 2001)

The Swiss engineer Heinz Isler succeeded in realizing almost 1400 shell structures with his method (Kotnik and Schwartz 2011). Eight most known shell structures with different geometries that were built and reused by him in the majority of his shell structures were selected and remodelled for finite element analysis. Table 1 briefly introduces these structures. Isler used four different physical methods for modelling and form finding of shells which can be categorized as:

1. Rubber membrane method - in this method, inspired by the pillow, a rubber membrane was put under internal pressure, and consequently the shape of the membrane under pressure represented the desired shell. The shell 1 of Table 1 is obtained by this method.

2. Hanging textile method-in this method, soft materials like fabrics that have not any resistance against compression, bending, shear and torsion find their forms under different loads, such as their self-weight, only by their tensile resistance. This feature produces tension only forces in the form attained by fabric, that is converted to 'all compression' state under inverse configuration, which is the most suitable force for the masonry and concrete materials. Hence, Isler selected this technique using the shape of hanged soaked textile, formed under their self-weight (shell 4, 5).

3. Foam flow method-this method is inspired by observing the foam growth. The shells 6 and 7 are acquired by this approach (Isler 2002).

4. Shaking method-in the later years of his life, Isler was looking for a method of finding forms by shaking them; however, this method was not well illustrated and applied to form finding of any shell structure.

In addition to the data publicly available in the references on Isler's work, the geometric dimensions of the models were acquired either from the scan reports of the existing miniature shell structures found in his workshop, or from the data of a number of existing blue print drawings of shells (Fig. 2) 
Table 1 Selected Isler's shell structures, unit: $\mathrm{m}$

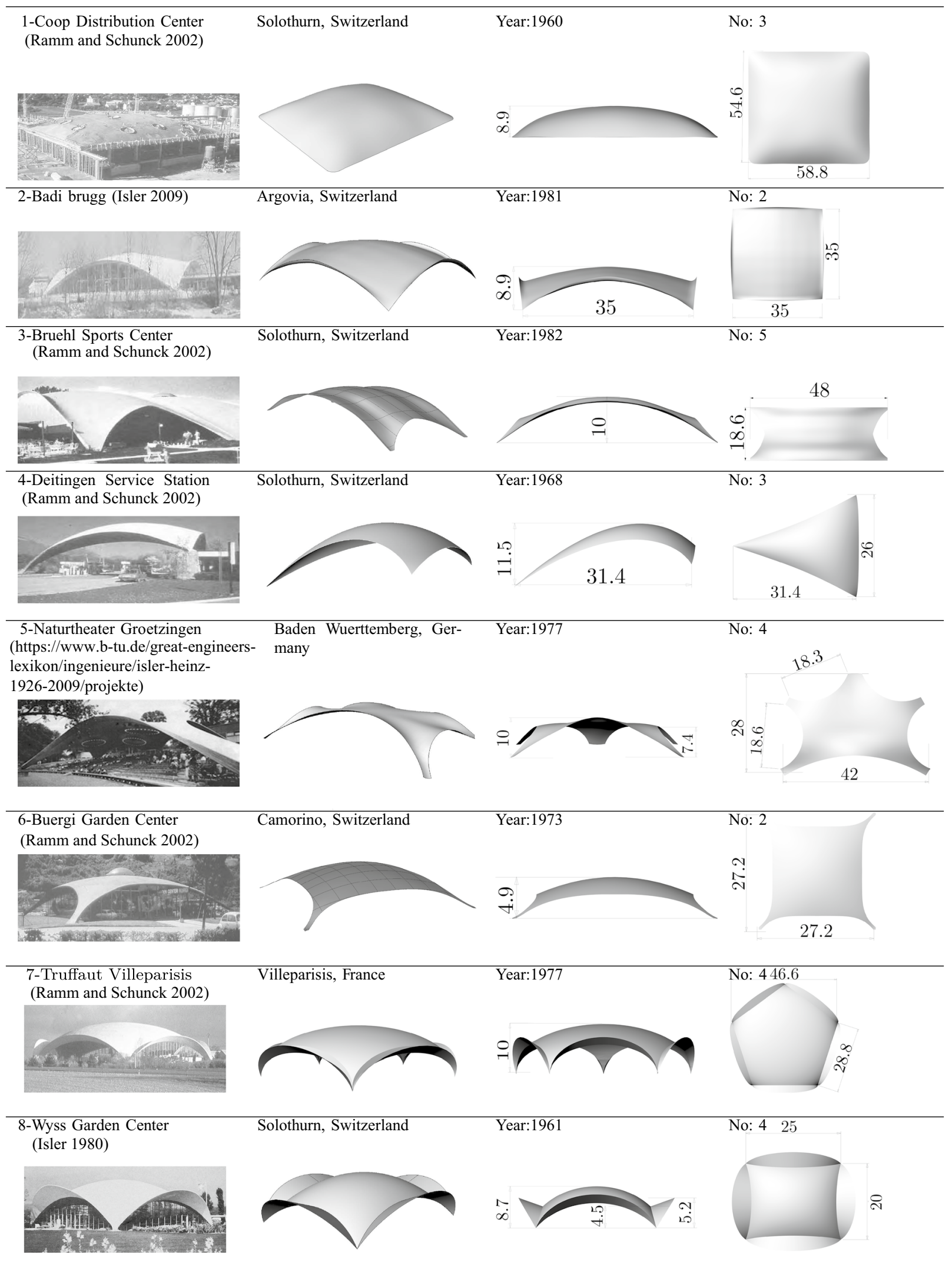

No: is the minimum number of all different arches required for re-modeling process. Each of the arches, which are embedded in the shells, might be repeated in a shell with changing dimentions 
(Stefan 2001). These data include the thickness, the span and the height dimensions of shells and plenty of pictures from different angles (Isler 2009). For the shell 7, different heights were mentioned in the references, the most probable height was assumed for it. The thicknesses of the shells varied and the assumed values here are based on the most probable ranges found in the relevant references, for instance for shell 1 thickness $(\mathrm{t})$ varied in the range $(15 \leq t \leq 19 \mathrm{~cm})$ (Christian and David 2012), for shell $5(8 \leq t \leq 15 \mathrm{~cm})$ (Abtin Baghdadi 2013), for shell 3 ( $t \sim 9 \mathrm{~cm})$ (Isler 2002), shell 8 ( $\leq 6$ $\mathrm{cm})$ (Chilton 2010). However, for the analysis, the thickness of all shells $t=15 \mathrm{~cm}$ is assumed for the reason of comparison. Obviously, in an individual assessment, the thickness of shell must be considered exactly or similar to the original structure. These thicknesses vary through the shell and have a direct relation with the vertical-distribution of these geometries. Essentially, under gravity loads, the forces increase from top to down of the shells, on the other hand, the forces above any level, are in balance with the forces below it which are obtained by multiplying the level forces with the perimeter and the thickness of the shell. Hence, in the absence of horizontal loads, the optimal design of shells, inherently demands for a variation of thickness with the height of the shell, which in turn leads to accurate and exact data for non-prismatic geometries. On the other side, however, assuming a uniform thickness inspired from real geometries to acquire a general account of force and stress distribution, is unavoidable.

The pictures taken straightly at frontal angles of all the arches enabled the deduction of extra geometrical data. Consequently, a large number of arch lines could be reconstructed, in their exact locations and angles. Hence, the arch frames were reconstructed for the entire shells, and finally, the surfaces located between the arches restored the full shape of the shells.

The main steps for modelling of shell structures are as follows:

1. Frontal straight angle pictures (2-6) from each arch of the shell were sent to AutoCAD, and their spans were rescaled as required.

2. Depending on the size of the arch, adequate number of nodal points was selected on a hypothetical curve which assisted finding the coordinates of the points for the next stage.

3. To draw the shape of the model, the xyz-coordinates of its grid points in plan or 3D space were transferred from AutoCAD to MATLAB.

4. Then, in MATLAB, the data were interpolated and a unique function was defined for each arch, and the height of the arches were rescaled which resulted in smoothed new $x y z$-coordinates for each arch with a symmetric smooth shape. At this step, a number of trigonometric

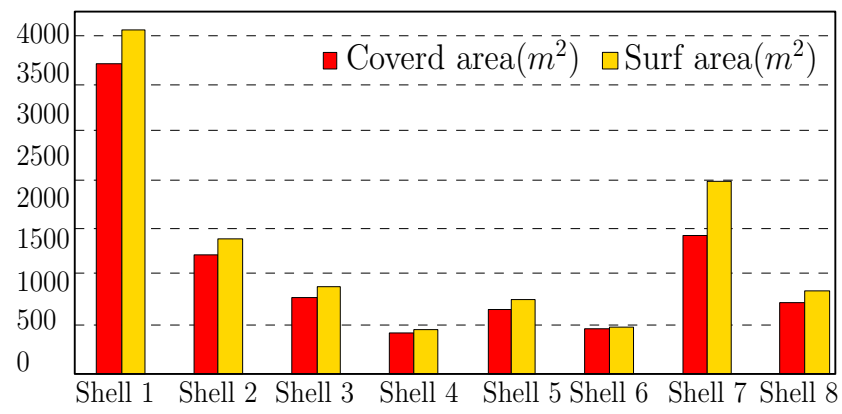

Fig. 3 Shells' area

functions were used to find out similarities between the arches.

5. The data of the curves were transferred to the Excel. Later, in Rhino, with the help of Grasshopper these nodes were converted into arches.

6. In Rhino, the bundle of all essential arches of each shell are replaced with their corresponding plan and the related angle.

7. Finally, the interconnection pattern is produced in Rhino, which readily could be conveyed into finite element software.

\section{Discussion}

\section{Evaluation of geometrical properties of shells}

The bar charts of Fig. 3 show the amount of surface areas of the shells together with the projected flat areas covered by them. The ratio of the " $\beta=$ surface-to-covered area" of a shell intriguingly is related to its " $\alpha=$ rise-to-span" ratio. The ratio $\alpha$ has the most influential role in optimizing the force flow in the shell. However, since a single "rise-tospan" ratio could not represent the spatial characteristics of a shell, consequently, such a relationship could not be defined simply.

Shell 1 covers the largest area and shell 4 covers the smallest area, while the shapes of shells 7 and 4 are attained by the flow and hanging method and have the maximum and the minimum ratios $\beta$, respectively. The source of difference in $\beta$ is the amount of the curvature of the shell's inflation. Figure 4 pictures the height of the shells together with their longest span, additionally, by considering both spans of the shells, the ratio $\alpha$ can be extrapolated and deduced. Shell 1 numerically has the largest span, which is placed on stiff boundary beams and columns, and is not structurally comparable with other shells. As a result, the shells 3 and 4 , with their unique shapes are genuine longest and highest shells, respectively, which have been found by second physical method. The ratio $\alpha$ for these shells on the average 


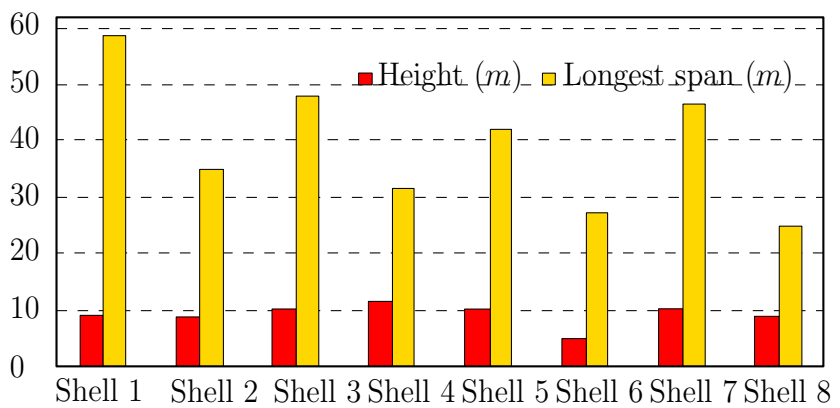

Fig. 4 Height and span of shells

is around 0.25 , while for the optimal arches, this ratio must be $[0.25,0.3]$. Thus, this parameter was satisfied for the shells, however, for shell 1 , which is obtained by the rubber membrane method this ratio is not fulfilled.

Thickness and height-to-span ratio are the most important parameters in optimizing shell's geometry, which is not uniform in these shells and the assumed values in this study are based on the ranges documented in references. In Isler's shells the average thickness is $10 \mathrm{~cm}$. But this is not always the case, and is related to his form finding method, in which the ratio $\alpha$ correlates with less thickness. For example, if the distance between lowest starting point and crown of each shell be the considered as its height (Table 1), shell 4 has the highest ratio $\alpha$ and lowest thickness while the contrary is true for shell number 1 .

Distribution of form in plan area is the matter of discussion in form finding, which can be compared with the Area Moments of Inertia (MOI) in which the thickness of the shells is not considered. Hence, to find the criteria, the distribution is divided by the area of the shells, and thus:

$I_{x}=\int\left(y^{2}+z^{2}\right) \mathrm{d} A$ and $I_{y}=\int\left(z^{2}+x^{2}\right) \mathrm{d} A$.

According to this formula, the symmetry of shells 2 and 6 with respect to $x$ and $y$ axis, shows the same amount of (MOI/ Area) in Fig. 5. There is an exception in shell 1, for which the average value of this factor is $\left.75 \mathrm{~m}^{2}\left(30 \leq I_{x, y} \leq 161\right)\right)$ while in $z$ direction it is about $140 \mathrm{~m}^{2}$. However, when the goal of optimization of shells is their performance under self-weight and pure compression, this factor cannot indicate any specific structural character, unless determination of their rigidities under vertical loads and assigning a specific number to them. Disability in dedicating a specific number to define the form is the biggest obstacle in form finding.

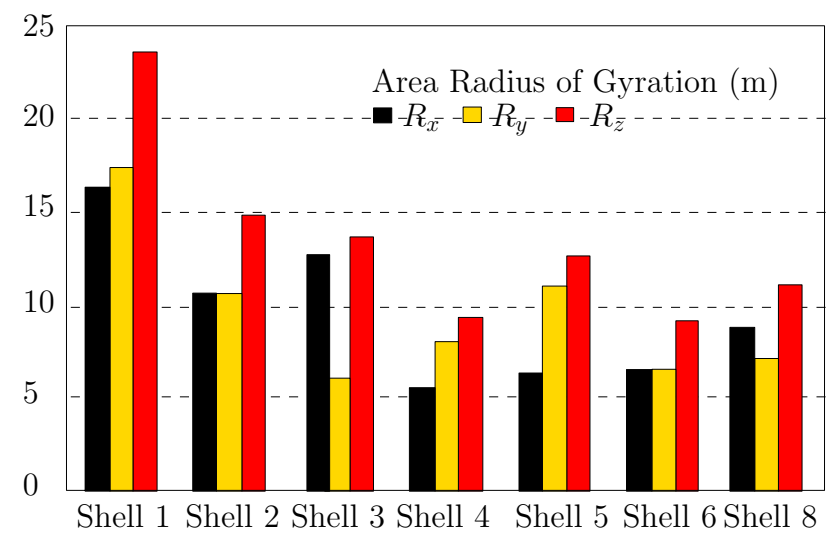

Fig. 5 Area moments of inertia/area

\section{Deformation of the shells}

Figure 6 shows the result of deformation of shell 6, under its own weight which is obtained using the software SAP2000 and Abaqus. The maximum negative displacement (towards gravitational forces) is $-1.76 \mathrm{~cm}$ which occurs at the middle of four edges. The displacement contours which are in the same direction as the gravitational loads somehow are measure of the stiffness of the shell in the gravitational direction as well. Figure 7 shows the deformations of shells 1, 2, 4 and 8 under their self-weights, where their extreme negative downwards displacements (towards gravity) are $-0.82 \mathrm{~cm}$, $-4.3 \mathrm{~cm},-18 \mathrm{~cm}$, and $-0.43 \mathrm{~cm}$, respectively. Within small regions near the supports of the shells positive (upwards) displacements (orange to red regions of Fig. 7) appear, but for shell 1, whose supports are continuous over the boundaries, the positive displacements occupy more sizeable area.

For the shells 1,2, and 8, the largest negative deformation occurs in the inner (blue) regions of the surface; nonetheless, for shell 4 , it occurs at the middle of the free edge of the shell. The deformations of Figs. 6 and 7, reveal that in general, Isler's shells have high rigidity, to elaborate further, the deformations of these shells vary in the range of $[-18,+10]$ $\mathrm{cm}$, where these belong to shell 4 , and thus it has the least stiffness among all eight shells. On the contrary, the shell 8 has the most robust geometry. However, in shell 8, the location and magnitude of the largest displacement will alter drastically due to change in the stiffness of its supports; in other words, the deformations of this shell are highly sensitive to its support conditions. Broadly, the shell structures found by hanging method have lowest stiffness in comparison to the other methods of form finding.

\section{Stresses of shells}

The criteria of von Mises stress that is based on the energy concept and incorporates the shear deformations as well 

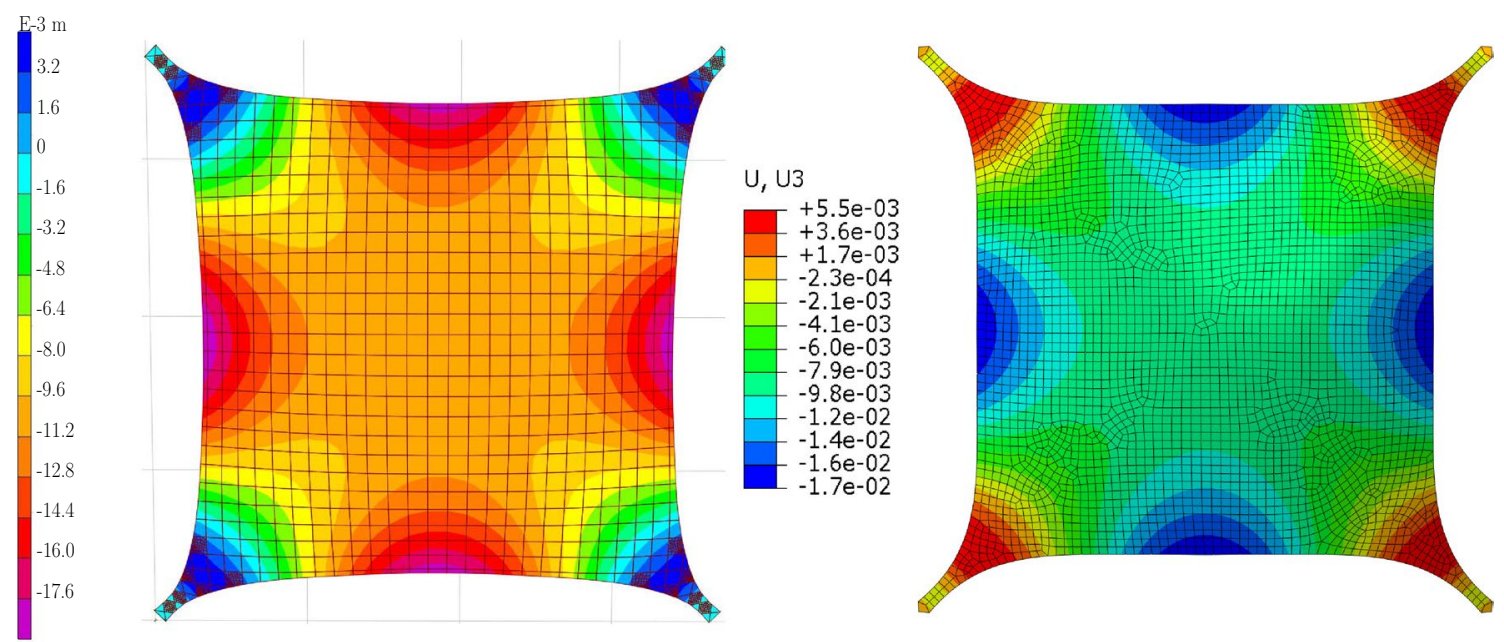

Fig. 6 Deformations of shell 6 (in metre, towards gravity), SAP2000 (left) and Abaqus (right)
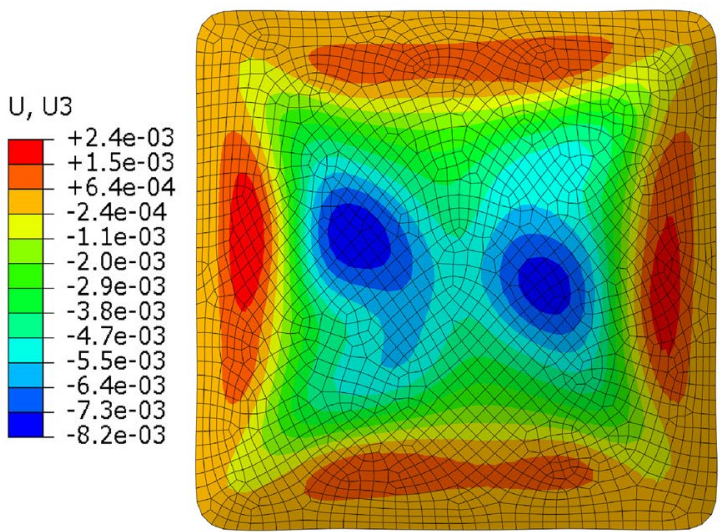

\section{$\mathrm{U}, \mathrm{U} 3$}
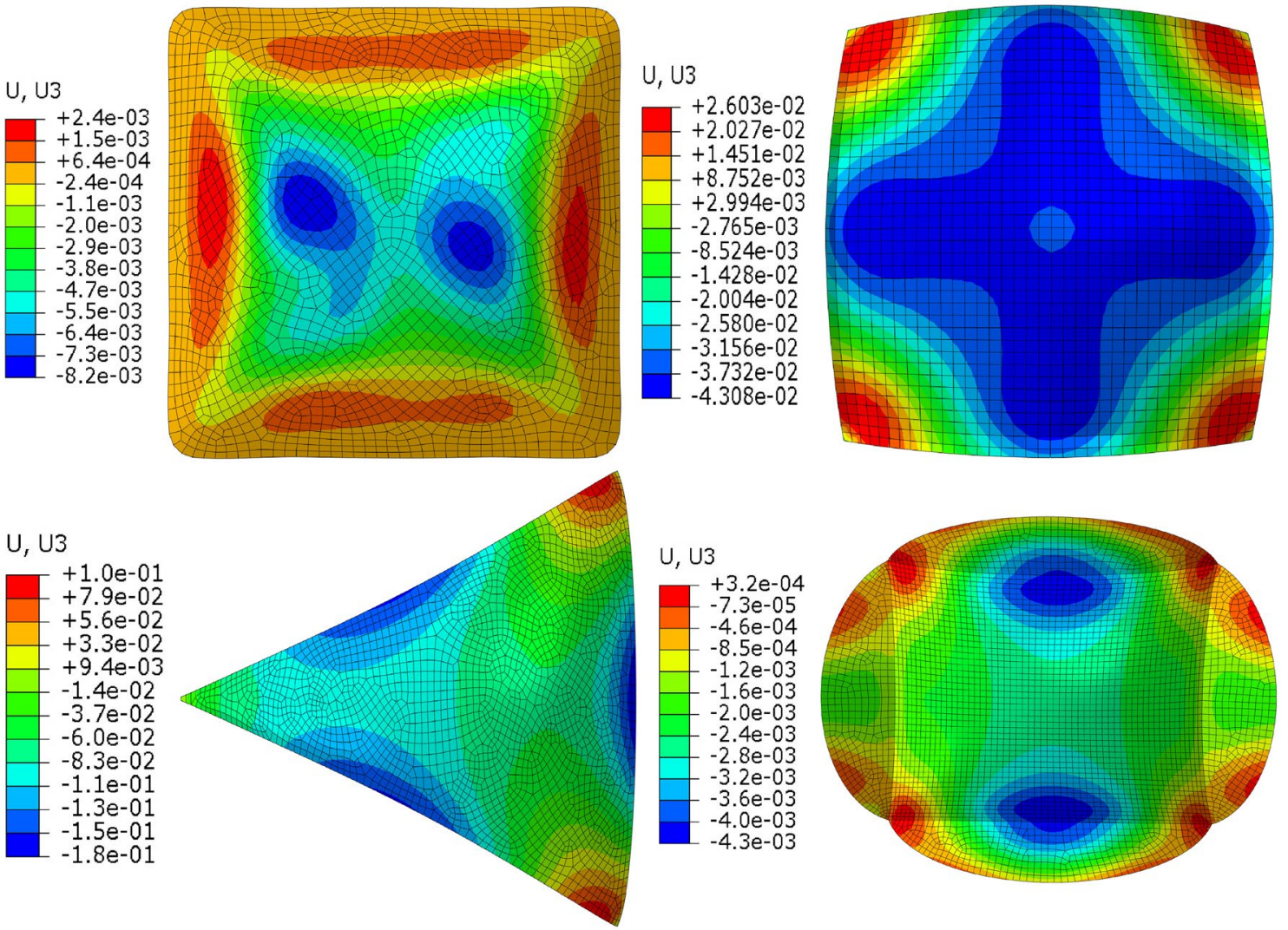

Fig. 7 Deformations of shells 1 (top-left), 2 (top-right), 4 (bottom-left), and 8 (bottom-right), Abaqus (in metre, towards gravity)

as various stress components is selected for the purpose of comparison of stresses in various shells, which is also the most common stress measure among different software. In Fig. 8, the stress distribution of shell 4, in software SAP2000 and Abaqus are plotted next to each other to show their close similarities. Several decades of satisfactory performance of Isler's shell structures signifies the fact that their stresses should not have exceeded the allowable limits of concrete $\mathrm{C}-28$ having the nominal compressive strength of $2800 \mathrm{t} / \mathrm{m}^{2}$ except the support points where, 


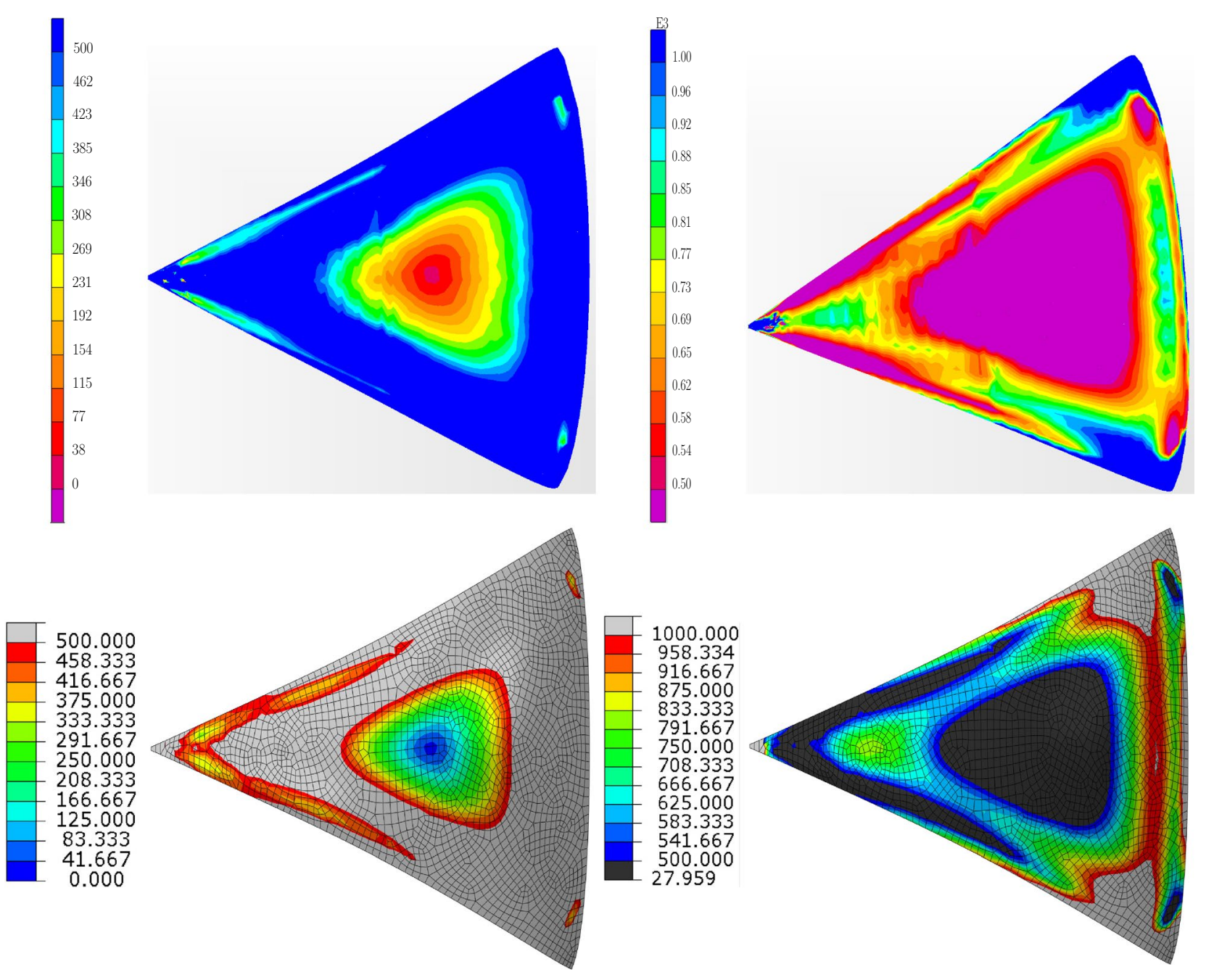

Fig. 8 Shell 4: von Mises stress from 0 to 500 (top-left) 500 to 1000 (top-right) (t/m²), in SAP2000 and Abaqus (bottom), in which since the details of supports are not discussed the amounts of stress in these zones are not displayed

an increase of stress is observed and Isler increased their dimensions drastically. Figure 9, represents the von Mises stress distribution for shells 2, 3, 6 and 8, under their selfweight. The large part of shell 2 , has the low stress in the range of $34-198 \mathrm{t} / \mathrm{m}^{2}$, nevertheless, there are some small patches of stress in the range of $525 \mathrm{t} / \mathrm{m}^{2}$ near the support lines, and finally in the tiny areas near the four corners the stress amounts to $2394 \mathrm{t} / \mathrm{m}^{2}$. The other shells have markedly lower stress concentrations near the support points, and their values do not exceed the limits 1511 , 1313 and $1623 \mathrm{t} / \mathrm{m}^{2}$, for the shells 3,6 , and 8 , respectively. With the exception of the supports, it can be stated that the amount of stress in shells 1-8 are normally less than $500 \mathrm{t} / \mathrm{m}^{2}$, and near the apex of the shells, it is about zero. Assessment of the stresses of shell 2 shows the average stress of $334 \mathrm{t} / \mathrm{m}^{2}$ along the diametrical curves connecting the opposite supports, while the numerical modelling and Isler's simple calculations give rise to a stress lower than the concrete nominal strength for that (Chilton and Isler 2000), but both of them are lower than the concrete nominal strength $\left(2800 \mathrm{t} / \mathrm{m}^{2}\right)$.

A comparison reveals that shells 1 and 8 have the first and second highest strength, and then with the exception of the supporting legs there are shells 6 and 4, respectively. Shell 4 can be considered as the most optimal shell because of its optimal use of material, and shell 1 is the least optimal. The high strength indicates that these two shells among all eight shells have minimum strain energy (or deformation) regarding the span and areas covered by them, while the most optimum shell is more flexible, along with having the least eccentricity $(e)$. It can be stated that the shell forms found by the physical hanging method have low deviations from the optimal performance. Another interesting point about Isler's shells is their plastic behaviour during yielding of reinforced concrete. By increasing the loads, within the Riks analysis in Abaqus, in most of them plastic hinges 


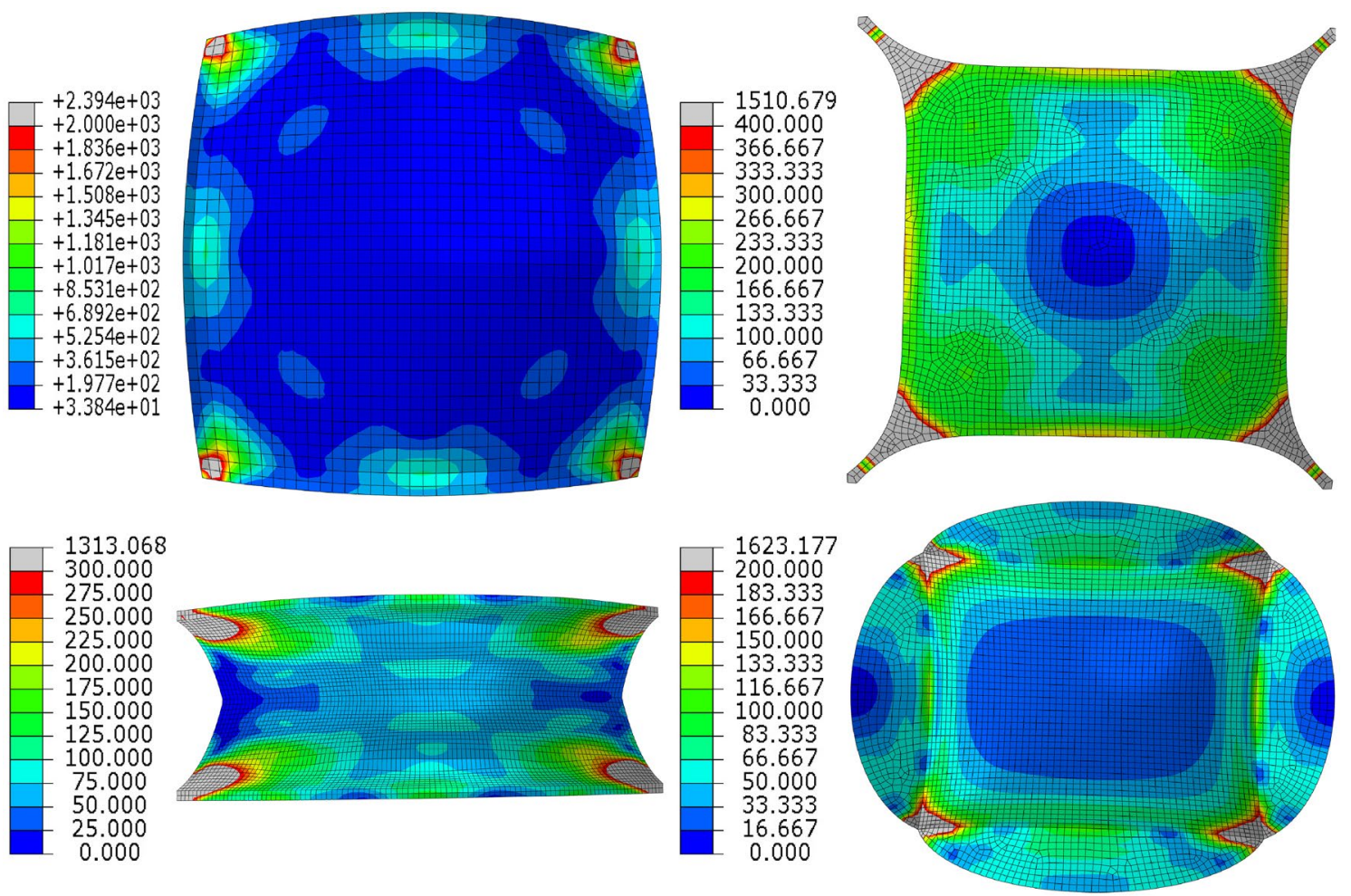

Fig. 9 von Mises stress of shells 2, 3, 6 and 8 in Abaqus in $\mathrm{t} / \mathrm{m}^{2}$

initiate from the lowest levels and then spread and move up to the top parts of the shells, to elaborate further, plastic hinges forms at sections of the same level without collapsing sideways or locally (increasing the membrane stress), and this phenomenon is a notable point in the ultimate behaviour of shells.

\section{Buckling and frequency analysis}

This section evaluates the resistance of Isler's shells to buckling. The buckling and frequency analysis of shells require modelling of exact support details, however, only for the purpose of a comparative evaluation of shells, identical support conditions are assumed for them. Under these assumptions, shells number 1, 7 and 8 had acceptable buckling loads, whereas in other shells, particularly in shells obtained by the hanging method, such as shell 3, this type of failure was probable. Additionally, the buckling is calculated for (uniformly distributed) dead load only, however, by adding snow or wind loads, due to their anti-symmetrical nature, the shells will be more susceptible to buckling. In some shells such as shell number 1 , which is surrounded by supports all around, and has a relatively higher thickness to span ratio, buckling is not a prime concern. The buckling failure was Isler's major concern in design of shells that is why in some projects he added extra elements such as cables near the
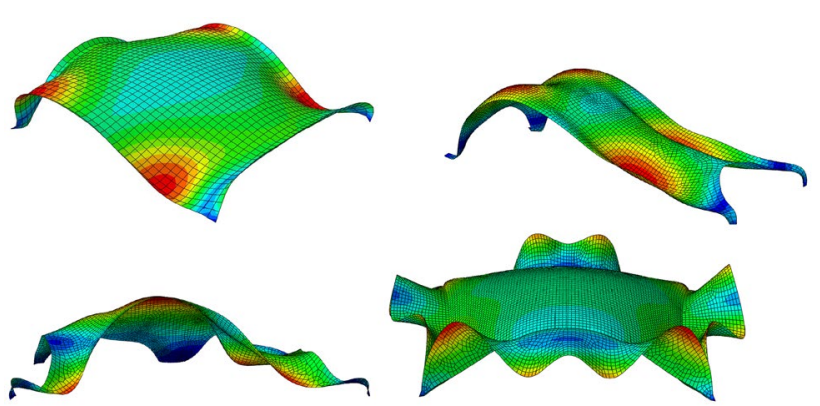

Fig. 10 First mode shapes of shell 2 (top-left), 3 (top-right), 5 (bottom-left) and 8 (bottom-right)

supports as a source of pre-stressing and restraining the supports from moving and highly strengthened them. In addition to buckling, the modal analysis was performed for the shells, which indicated that the highest eigenvalue and the lowest period time belong to shells $1,7,8$ and 6 , respectively. The analysis also shows a sequence of symmetrical mode shapes in almost all of the shells. Figure 10, shows different modal behaviour of shells 2, 3, 5 and 8 . 

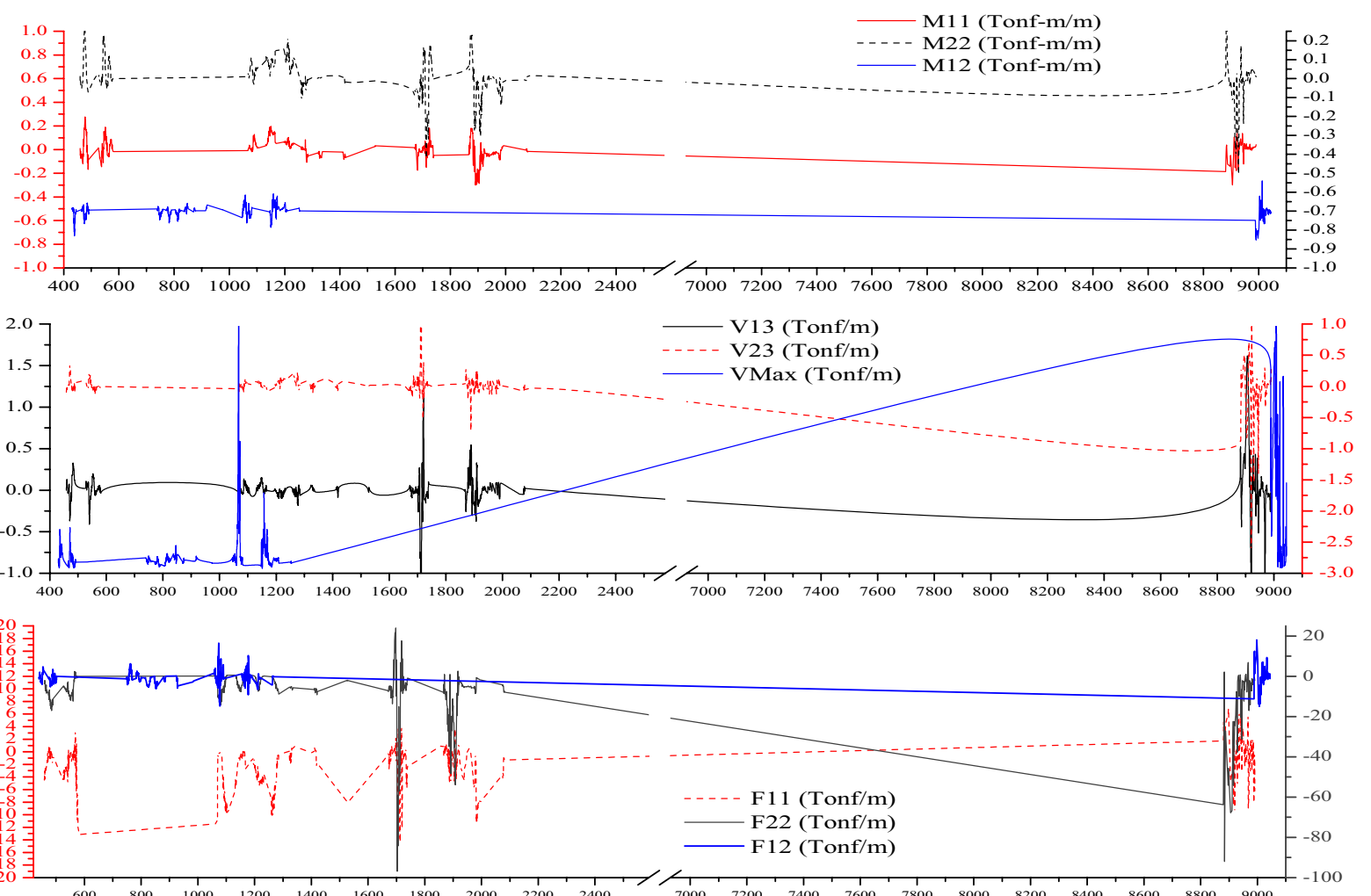

Shell 3 mesh number

Fig. 11 Shell 3 internal forces (bending moment) $(M)$, shear $(V)$ and axial load $(F)$ for each mesh of shell $(t, m)$

\section{Internal forces}

Since, considering the forces gives a better overview about the behaviour of shells, therefore, in addition to von Mises stresses which were given in "Stresses of shells" section, this section deals with the forces in shells. To clarify the acts of the shells the axial load $(F)$, shear $(V)$ and bending moments $(M)$ in all directions of each shell are presented. It is seen from Figs. 11 and 12 that the amount of bending moment, and shear force compared to axial load has a low value. This observation could be related to the pressure line theory, in which the assumed nodes of an arch (or shell) and their related eccentricity $(e=M / F)$, leads to the so called pressure line (or area). This means that the hypothetical form (arch or shell) complies with the Isler's shell forms.

$\sum_{i}^{n} e_{i}=\left|\frac{M_{i}, V_{i}}{F_{i}}\right| \rightarrow 0$

This type of force and ratio typically occurs in all the modelled shells and presents the same type of behaviour. For instance in shell $3(t, m)-95 \leq F_{11}, F_{22} \leq 20$ while $-3.0 \leq M_{11}, M_{22}, V_{13}, V_{23} \leq 1$ and for shell 4
$-110 \leq F_{11}, F_{22} \leq 40$ while $-5 \leq M_{11}, M_{22}, V_{13}, V_{23} \leq 2.2$. In all the shells, the maximum amount of shear and bending moments (= eccentricity) belongs to the supports which are not in the scope of form finding process.

It can be shown that the average eccentricity in eight of mentioned shells is around 0.125 , whereas in shell 1 , which was achieved by Isler's first method, it is limited to 0.015 . The source of this deviation of eccentricity from zero might be the method of experiment and/or approximations in reverse engineering. The low eccentricity verifies again the accuracy of all steps of back engineering and analysis in this study.

This calculation also shows the accuracy of Isler's method in form finding of shells, and his special ability and experience in optimal designing of shell structures.

Where, $M_{11}$ and $M_{22}$ are direct moments per unit length at element mid-surface on faces 1 and 2 , respectively. $V_{13}$ is the out of plane shear per unit length at element mid-surface on face 1 in direction 3 , and $V_{23}$ is the out of plane shear per unit length at element mid-surface on face 2 in direction 3. $F_{11}$ is the direct force per unit length at element mid-surface on face 1 and direction $1, F_{22}$ is the direct force per unit length at element mid-surface on face 2 and direction 2. 

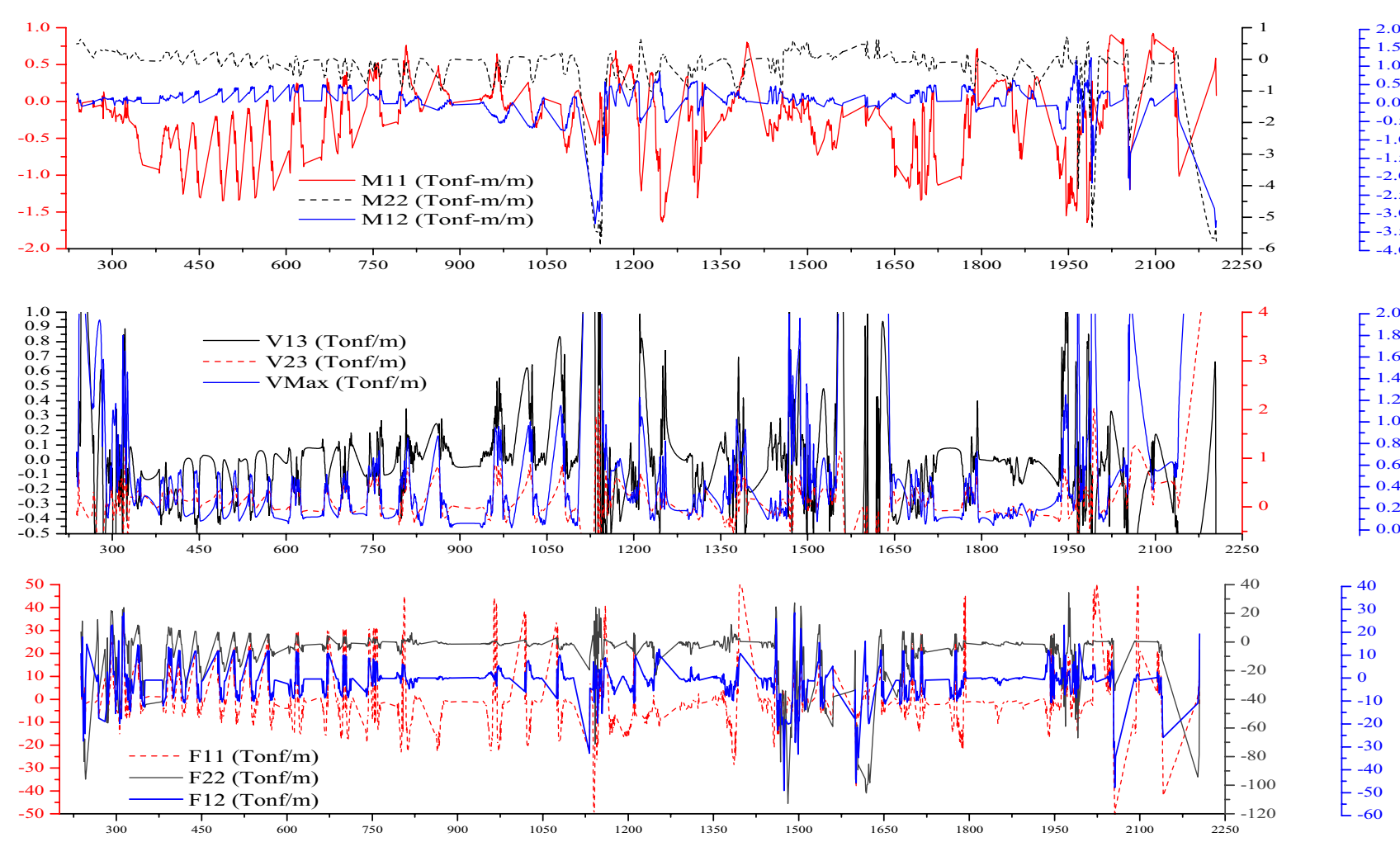

Shell 4 mesh number

Fig. 12 Shell 4 internal forces (bending moment) $(M)$, shear $(V)$ and axial load $(F)$ for each mesh of shell $(t, m)$

\section{Concluding remarks}

In this research eight of Heinz Isler's shells as the last generation of designers mimicking the natural structural shell forms are back engineered and assessed. The required data for remodelling of structures is generated by gathering numeric and visual data from various references and processing within several software packages. All care is taken to reconstruct the numerical models with a high degree of conformity to the Isler's shells, however, the possibility of a degree of deviation from the real 'as built' shells of Isler, could not be ruled out. Therefore, in view of this fact, one might consider the analysed models as 'approximations to true Isler's shells', and hence, the conclusions should be observed with reservations. Optimal performance of Isler's shells and their minimum eccentricity confirms his experience, special and gifted ability in form finding of shells. The vertical deformation of shells relative to their span is low, which is an indication of the fact that their load transmission is in membrane rather than in flexural form. This feature is the outcome of appropriate sharing of axial force, as well as incorporating suitable eccentricity which can be utilized in form finding and shell optimization calculations. On the other hand, Isler's shell structures exhibited bending moments, though not at a high degree compared to their axial loads, which applies to almost all practical approaches searching optimal forms. Regarding the buckling behaviour, Isler has stiffened the shells prone to instability and buckling. However, by and large, the buckling behaviour of the shells is unsatisfactory as compared to their other structural aspects. The differences between Isler's three form finding methods associated with the shell structures illustrates that the hanging method is superior to the other methods of shell optimal form finding with consideration to stiffness, concrete allowable stress and amount of material. With regards to these parameters the Deitingen service gas station (shell 4) with its unique form can be considered as the most optimal form whose architectural and structural features was not repeated by Isler in any structures even in decades since then.

Open Access This article is distributed under the terms of the Creative Commons Attribution 4.0 International License (http://creativeco mmons.org/licenses/by/4.0/), which permits unrestricted use, distribution, and reproduction in any medium, provided you give appropriate credit to the original author(s) and the source, provide a link to the Creative Commons license, and indicate if changes were made. 


\section{References}

Adriaenssens S, Block P, Veenendaal D, Williams C (2014) Shell structures for architecture: form finding and optimization. Taylor \& Francis, London

Aichtal-Grötzingen Baden-Württemberg (1977-1978) "BTU Cottbus-Senftenberg, Link zur Startseite, Theater in Grötzingen", Deutschland. https://www.b-tu.de/great-engineers-lexikon/ingen ieure/isler-heinz-1926-2009/projekte

Akhavan LMR, Tahooni S (1996) Structural analysis. Tehran Polytechnic University, Tehran

"Auf den Spuren der Schalenpioniere bis zu den Pionieren der robotergesteuerten Architektur", des Instituts für Tragwerksentwurf (ITE) und des Instituts für Industriebau und Konstruktives Entwerfen (IIKE), Seminarexkursion "PROTOFORM" (2013)

Borgart A, Eigenraam P (2012) Scanning in 3D and analysing the models of Heinz Isler, the preliminary results , IASS-APCS 2012: from spatial structures to space structures, Seoul, South Korea, 21-24 May

Brew JS, Lewis WJ (2007) Free hanging membrane model for shell structures. Int J Numer Methods Eng 71(13):1513-1533

Chilton J (2010) "Heinz Isler's infinite spectrum": form-finding in design. Archit Des 80:64-71

Chilton J, Chuang CC (2017) Rooted in nature: aesthetics, geometry and structure in the shells of Heinz Isler. Nexus Netw $\mathbf{J}$ 19(3):763-785

Chilton J, Isler H (2000) Heinz isler. Thomas Telford, London

Christian L, David B (2012) “Autobahnraststätte Deitingen-Süd-Schalentragwerk von Heinz Isler", Dozenten: Dr. Christoph Schindler, Kyeni Mbiti

Emami N (2015) Structure and daylighting performance comparisons of Heinz Isler's roof shell based on variations in parametrically derived multi opening topologies, IASS 2015. Future Visions Amsterdam

González G, Samper A, Herrera B (2018) Classification by Type of the Arches in Gaudi's Palau Güell. Nexus Netw J 20(1):173-186
Heristchian M, Baghdadi A, Ameri S (2014) Elastic and plastic buckling of steel arches under in-plane loadings. In: Fourth national Iranian conference on spatial structures, Tehran

Isler H (1980) Objekttyp: Article, Heinz Isler Peojects. http://www. library.ethz.ch/en/

Isler H (2002) Heinz Isler, Schalen: Katalog zur Ausstellung. vdf Hochschulverlag AG

Isler H (2009) International database for civil and structural engineering. https://structurae.net/

Kotnik T, Schwartz J (2011) The architecture of heinz isler. J Int Assoc Shell Spatial Struct 52(3):185-190

Maurer T, O'Grady E, Tung E (2013) Inverse hanging membrane: the Naturtheater Grötzingen. Evolution of German shells efficiency in form

Neuhäuser S (2014) "Untersuchungen zur Homogenisierung von Spannungsfeldern bei adaptiven", Schalentragwerken mittels Auflagerverschiebung

Ramm E, Mehlhorn G (1991) On shape finding methods and ultimate load analyses of reinforced concrete shells. Eng Struct 13(2):178-198

Ramm E, Schunck E (2002) Heinz Isler: Schalen: Katalog zur Ausstellung, vdf Hochschulverlag an der ETH

Sprague TS (2013) "Beauty, versatility, practicality": the rise of hyperbolic paraboloids in post-war America (1950-1962). Constr Hist 28:165-184

Stefan B (2001) Zuchwil, Gartenstrasse 32, GartenHaus Wyss AG

Timoshenko SP, Woinowsky-Krieger S (1959) Theory of plates and shells. McGraw-hill, New York

Publisher's Note Springer Nature remains neutral with regard to jurisdictional claims in published maps and institutional affiliations. 